\title{
Utilization of renewable durian peels for biosorption of zinc from wastewater
}

\begin{abstract}
Durian peel is among the renewable biomass wastes abundantly available in Malaysia. An implication of untreated biological materials for biosorption process was intensively reported, that prioritize our work towards sorbent modification. The biosorption potentials of hydrochloric acid $(\mathrm{HCl})$ modified durian peels (HAMDP) for removal of $\mathrm{Zn}$ (II) from simulated wastewater was investigated. Characterization of HAMDP was performed by ATR-FTIR, SEM and BET. Spectroscopic studies showed the predominant contributors for $\mathrm{Zn}$ (II) biosorption on HAMDP is attributed to hydroxyl, carbonyl, carboxyl and amides groups. Batch adsorption studies revealed optimum conditions of $\mathrm{pH} 8,0.5 \mathrm{~g}$ biosorbent dose, $4 \mathrm{~h}$ contact time and reaction temperature of $313 \mathrm{~K}$. Nonlinear isotherm models suggested applicability of Tempkin and Langmuir models at $313 \mathrm{~K}$. The Langmuir maximum adsorption capacity was $36.73 \mathrm{mg} / \mathrm{g}$. Kinetic studies revealed applicability of pseudo-second-order model. Webber-Morris model indicated possible role of diffusion of $\mathrm{Zn}$ (II) within the particles of HAMDP during the sorption process. Freundlich constant and activation energy values confirmed the physical nature of the process. Thermodynamic studies indicated that the process is exothermic and spontaneous. Regeneration studies depicted that HAMDP is economically viable. Conclusively, $\mathrm{HCl}$ served two significant purposes, namely; a good modification reagent and best eluent in $\mathrm{Zn}$ (II) recovery. Therefore, HAMDP is relatively effective, efficient, economical and most importantly "renewable and sustainable" biosorbent for Zn (II) removal from wastewater.
\end{abstract}

Keyword: Biosorbent reusability; Biosorption; Durian waste; Heavy metals; Modification; Wastewater treatment 